\title{
Development of Electron Bernstein Emission Diagnostic for Heliotron $\mathbf{J}^{*)}$
}

\author{
Kazunobu NAGASAKI, Yuji NAKAMURA ${ }^{1)}$, Sohei KAMIOKA ${ }^{1)}$, Hiroe IGAMI $^{2)}$, \\ Francesco VOLPE ${ }^{3)}$, Torsten STANGE ${ }^{4}$, Kinzo SAKAMOTO, Hiroyuki OKADA, \\ Takashi MINAMI, Shinichiro KADO, Shinji KOBAYASHI, Satoshi YAMAMOTO, \\ Shinsuke OHSHIMA, Gavin WEIR, Shigeru KONOSHIMA, Naoki KENMOCHI ${ }^{1}$, \\ Yoshiaki OTANI ${ }^{1)}$, Yasuo YOSHIMURA ${ }^{2)}$, Nikolai MARUSHCHENKO ${ }^{4)}$ and Tohru MIZUUCHI \\ Institute of Advanced Energy, Kyoto University, Gokasho, Uji 611-0011, Japan \\ ${ }^{1)}$ Graduate School of Energy Science, Kyoto University, Gokasho, Uji 611-0011, Japan \\ ${ }^{2)}$ National Institute for Fusion Science, Toki 509-5292, Japan \\ ${ }^{3)}$ Applied Physics and Applied Mathematics, Columbia University, New York, USA \\ ${ }^{4)}$ Max-Planck-Institut für Plasmaphysik, IPP-EURATOM Association, D-17491, Greifswald, Germany
}

(Received 25 November 2015 / Accepted 6 May 2016)

Electron Bernstein Emission (EBE) diagnostic system using Ordinary $(\mathrm{O})$ - eXtraordinary $(\mathrm{X})$ - Bernstein (B) mode conversion process has been developed for determining electron temperature profiles at overdense plasmas in a helical-axis heliotron device, Heliotron J. Ray-tracing calculation results show that the O-X mode conversion window is accessible with a current ECH/ECCD launcher and transmission system. The EBW radiation from the core region is expected to be measurable by adjusting the magnetic field strength. Effect of density fluctuation on O-X mode conversion efficiency is also discussed. Based on the calculations, a multi-channel radiometer of Ka-band range has been designed, assembled and tested.

(c) 2016 The Japan Society of Plasma Science and Nuclear Fusion Research

Keywords: electron Bernstein emission, diagnostic, ray tracing, radiometer, Heliotron J

DOI: $10.1585 /$ pfr. 11.2402095

\section{Introduction}

Electron cyclotron emission such as Ordinary $(\mathrm{O})$ and eXtraordinary $(\mathrm{X})$ modes are used for electron temperature diagnostic in magnetic fusion devices. Although the ECE diagnostic is reliable, there is an upper accessible density limit so called cut-off density. Electron Bernstein Waves (EBWs) are electrostatic waves propagating in hot magnetized plasmas at densities where the $\mathrm{O}$ and $\mathrm{X}$ modes are not accessible. The EBWs have advantage of no density limit and high optical thickness even at low electron temperature. These aspects, along with high localization of their emission, makes EBWs suitable for determining electron temperature profiles and the pitch angle of magnetic field at overdense plasmas [1]. While the EBWs can exist only in a plasma, they can be coupled to an external receiver by means of mode conversions. Of various schemes proposed, the Bernstein (B)-X-O process is attractive since they can be geometrically optimized by adopting a special line of sight [2-4].

In this paper, we show recent progress on theoretical study and diagnostic development for EBE measurement in a helical-axis heliotron device, Heliotron J, aiming at

author'se-mail: nakazaki.kazunobu.4x@kyoto-u.ac.jp

*) This article is based on the presentation at the 25th International Toki Conference (ITC25). measuring core electron temperature in overdense plasmas. The theoretical study of mode conversion and radiation position is described in Sec. 2, and a radiometer system for EBE diagnostic is shown in Sec. 3. Summary is given in Sec. 4.

\section{Ray Tracing Code for O-X-B Mode Conversion in Heliotron $\mathbf{J}$}

Heliotron $\mathrm{J}$ is a medium-sized stellarator/heliotron device [5]. The main device parameters are the plasma major radius $R=1.2 \mathrm{~m}$, the averaged minor radius $a=0.1$ $0.2 \mathrm{~m}$, the rotational transform $\iota / 2 \pi=0.3-0.8$, and the maximum magnetic field strength on magnetic axis, $B=$ $1.5 \mathrm{~T}$. The coil system is composed of an $L=1, M=4$ helical coil, two types of toroidal coils A and B, and three pairs of vertical coils. Here $L$ and $M$ denote the poloidal and toroidal pitch numbers, respectively. Accessing the fundamental EBE by means of B-X-O conversion requires the plasma densities over around $1 \times 10^{19} \mathrm{~m}^{-3}$, which are routinely produced in the Heliotron $\mathrm{J}$.

In toroidal geometries, an incident O-mode launched from the low field side can be converted into a slow $\mathrm{X}$ mode at the O-mode cut-off layer if the O-mode is injected at the optimal angle oblique to the magnetic field. The optimal angle at the O-mode cut-off is given by 


$$
\cos \theta^{\text {opt }}=\sqrt{\frac{Y}{1+Y}},
$$

where $\theta$ is the angle that the magnetic field makes with the wave vector, $Y=\omega_{\text {ce }} / \omega, \omega_{\text {ce }}$ is the electron cyclotron angular frequency and $\omega$ is the wave angular frequency.

Before studying EBW, we investigate the viewing angle for O-X mode conversion by using a ray tracing code, TRAVIS code [6]. The TRAVIS code was developed for $\mathrm{ECH} / \mathrm{ECCD}$ and ECE diagnostic in arbitrary 3D magnetic configurations. The magnetic configuration is provided by the $3 \mathrm{D}$ equilibrium code VMEC. The code is used through an especially designed graphical user interface, which allows the preparation of input parameters and viewing results in convenient (2D and 3D) form. This interface makes it rather easy to find out the parallel and perpendicular refractive indices at cut-off positions. The mode conversion efficiency is given as

$$
\begin{aligned}
& T\left(N_{y}, N_{z}\right)= \\
& \exp \left\{-\pi k_{0} L_{n} \sqrt{\frac{Y}{2}}\left[2(1+Y)\left(N_{z, \mathrm{opt}}-N_{z}\right)^{2}+N_{y}^{2}\right]\right\},
\end{aligned}
$$

where $N_{z, \mathrm{opt}}^{2}=\cos ^{2} \theta^{\mathrm{opt}}=Y /(1+Y)$, and $L_{n}$ is the density scale length [7]. Figure 1 illustrates the contour of $\mathrm{O}-\mathrm{X}$ mode conversion efficiency in viewing space for $35 \mathrm{GHz}$ frequency waves. The mode conversion window with more than $50 \%$ efficiency has an angle range of about $8 \mathrm{deg}$ and $3 \mathrm{deg}$ in the toroidal and poloidal directions, respectively, at electron density of $n_{\mathrm{e}}=2 \times 10^{19} \mathrm{~m}^{-3}$. Although the accessible toroidal angle in the experiment is limited by steering range of the antenna mirror and the port boundary, we expect that the conversion efficiency more than $50 \%$ is obtained at available viewing angles. As the mode conversion efficiency is a function of density scale length, the $\mathrm{O}$ $\mathrm{X}$ window is wider with an increase in electron density as shown in Fig. 1 (b), which would be beneficial for searching the $\mathrm{O}-\mathrm{X}$ conversion point.

A ray tracing code, KRAY, upgraded from B-RAY code [8], has been developed to calculate the ray trajectory of O-mode, X-mode and EBW for Heliotron J. New aspects on the KRAY code development, compared to the previous B-RAY code is that the magnetic coordinates given by the VMEC code were newly taken into consideration in the KRAY, while the magnetic field is given by filament coils and the flux surface is assumed elliptical in the poloidal cross-section in the B-RAY code. Note that the $\mathrm{O}-\mathrm{X}-\mathrm{B}$ calculation is applicable to EBE measurement via $\mathrm{B}-\mathrm{X}-\mathrm{O}$ mode conversion since the mode conversion process is reversible. The code solves the radiative transfer equations under the geometrical optics approximation. To calculate the ray trajectory, the three-dimensional magnetic field structure and flux surfaces of Heliotron $\mathrm{J}$ is taken into account, which are given by the VMEC code with finite $\beta$. The dispersion relation for the electromagnetic waves is given by a cold plasma approximation, and the dispersion
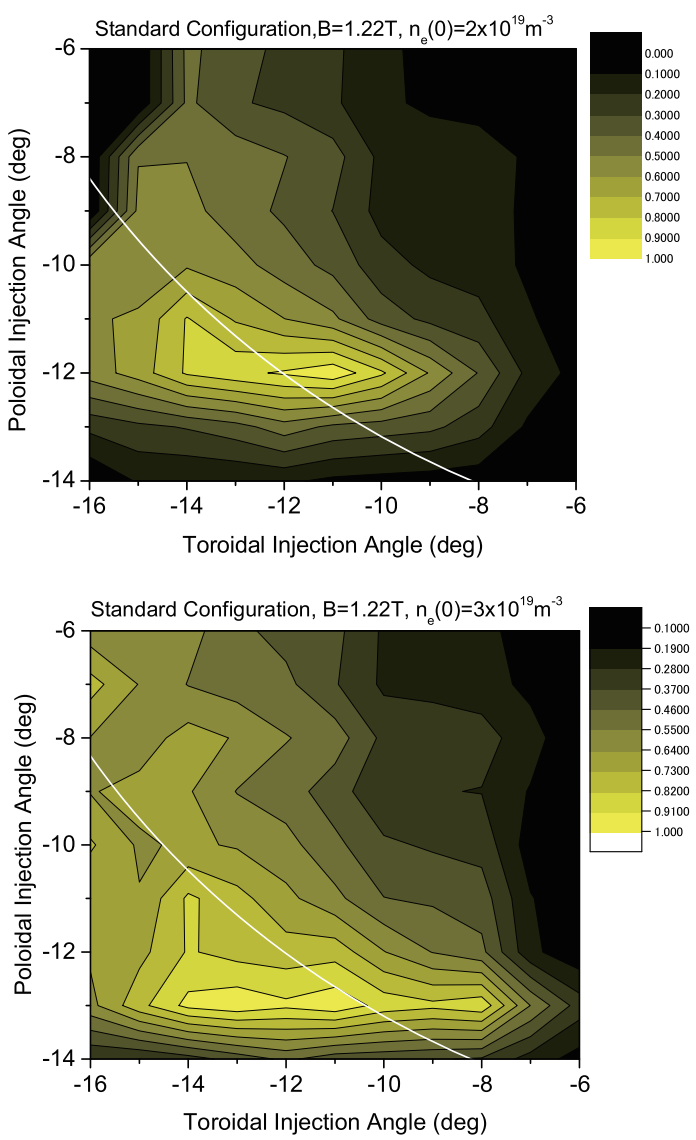

Fig. 1 Contour of O-X mode conversion window at $B=1.22 \mathrm{~T}$, (a) $n_{\mathrm{e}}(0)=2 \times 10^{19} \mathrm{~m}^{-3}$ and (b) $n_{\mathrm{e}}(0)=3 \times 10^{19} \mathrm{~m}^{-3}$. The white curved line denotes the port boundary.

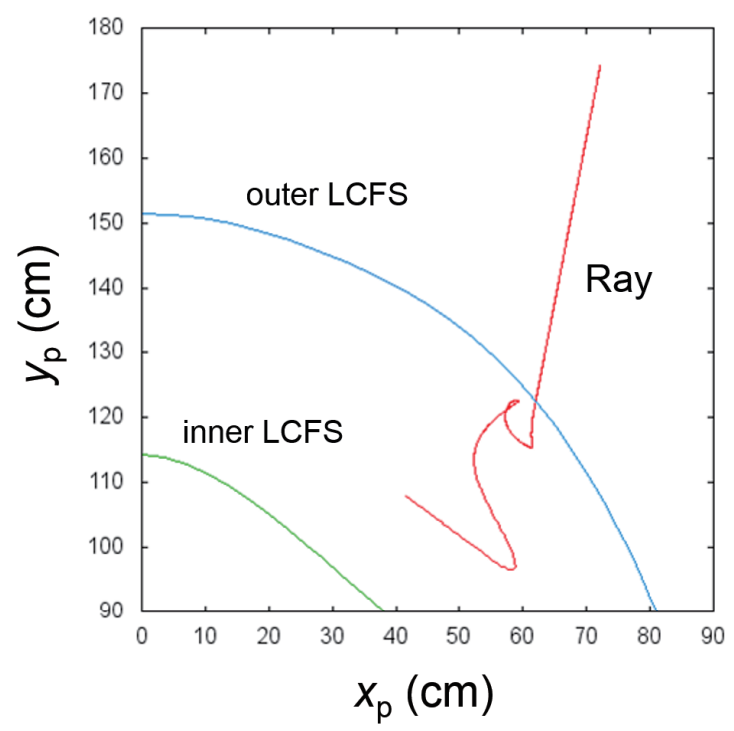

Fig. 2 Top view of ray trajectory in O-X-B mode conversion process in the Heliotron $\mathrm{J}$ standard configuration.

relation for electrostatic waves is given by a hot plasma approximation.

Figure 2 illustrates a top view of a ray trajectory in $\mathrm{O}-\mathrm{X}-\mathrm{B}$ mode conversion in the standard Heliotron $\mathrm{J}$ con- 


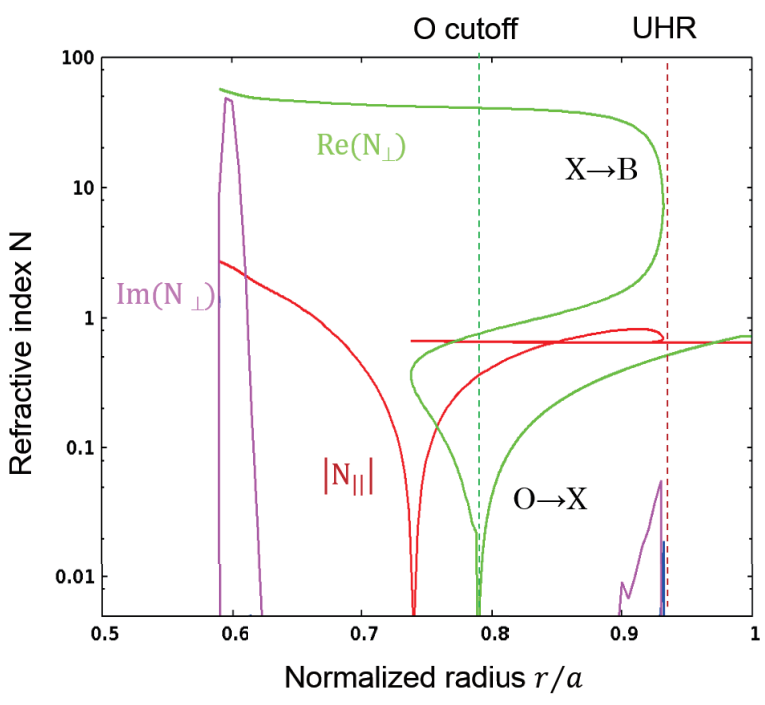

Fig. 3 Refractive indices as a function of plasma radius.

figuration, and Fig. 3 shows the refractive indices along the ray trajectory. In the KRAY code, the optimal viewing angle for $\mathrm{O}-\mathrm{X}$ mode conversion is searched as well as the TRAVIS code. We confirm that the $\mathrm{O}-\mathrm{X}$ window region estimated in KRAY agrees with that by TRAVIS code. The real part of perpendicular refractive index goes to zero at the O-mode cut-off, and then the O-mode is converted into the slow X-mode. After conversion, the X-mode propagates toward high-density region until it turns back and propagates toward the upper hybrid resonance (UHR) layer. When the X-mode approaches the UHR layer, the perpendicular component of the refractive index rapidly increases, resulting that the phase velocity of the wave slows down. In this process, the electric field of the X-mode begins to lie parallel to the wave vector, that is, the wave turns to be the EBW. The generated EBW propagates backward into the high-density region, and the imaginary part of perpendicular refractive index rapidly increases at the position where the Doppler shifted cyclotron resonance condition is satisfied, resulting that the wave energy is transferred to electrons. The EBW from the radiation position has the sign of $N_{\|}$opposite to that of the EBW traced from the UHR. According to the Doppler-shifted resonance condition, $\omega\left(1-N_{\|} v_{\|} / c\right)=\omega_{\text {ce }}$. the electrons with the opposite sign of $v_{\|}$resonate with the EBW when $N_{\|}$is reversed, resulting that the deposition position is the same as the radiation position. If the velocity distribution is asymmetric with $v_{\|}$, the deposition position may be different from the radiation position.

As shown in Fig. 4, the absorption position, which reflects the radiation position of the EBW, is located at core region if the magnetic field is properly chosen. As $\beta$ increases, the magnetic axis is Shafranov-shifted outwardly, affecting the radiation position. The radiation frequency to measure the core electron temperature is $37 \mathrm{GHz}$ at $1.20 \mathrm{~T}$, where the radiation position closest to the magnetic axis

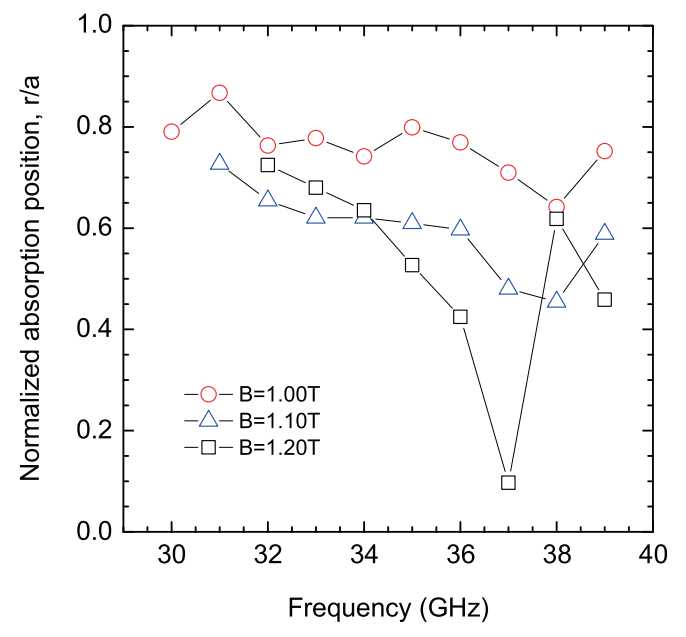

Fig. 4 Calculated absorption radius against wave frequency at $n_{\mathrm{e}}(0)=2 \times 10^{19} \mathrm{~m}^{-3}$.

is $r / a=0.1$. In the calculation, the radiation position is determined by the ray with optimum launching condition. Since the $\mathrm{O}-\mathrm{X}$ window has a finite area, the radiation profile is broader when we include the ray trajectories with non-optimum conditions. Accurate radiation profile will be studied in future work.

Density fluctuation gives rise to beam divergence, resulting in reduction in the $\mathrm{O}-\mathrm{X}$ conversion efficiency. Let us consider the fluctuation with a wave vector in the poloidal direction. With a statistic description of the cutoff surface roughness, the probability density function of the poloidal component $N_{y}$ is given as

$$
p\left(N_{y}\right)=\frac{\lambda_{y}}{\sqrt{2 \pi} \sigma_{x}} \exp \left\{-\frac{N_{y}^{2} \lambda_{y}^{2}}{\left(1-N_{y}^{2}\right) 2 \sigma_{x}^{2}}\right\}\left(1-N_{y}^{2}\right)^{-3 / 2},
$$

where $\sigma_{x}$ is the the fluctuation amplitude standard deviation, $\sigma_{x}=L \tilde{n}_{\mathrm{e}} / n_{\mathrm{e}}, \lambda_{y}$ is the poloidal correlation length [9]. The modified $\mathrm{O}-\mathrm{X}$ conversion efficiency, $T_{\text {mod }}$, including density fluctuation effect is written as

$$
T_{\text {mod }}\left(N_{z}\right)=\int_{-1}^{1} T\left(N_{z}, N_{y}\right) p\left(N_{y}\right) d N_{y} .
$$

Figure 5 shows the dependence of modified O-X mode conversion efficiency, $T_{\text {mod }}$, on the viewing angle. Here we assume that $\omega_{\text {ce }}$ is equal to $\omega$. The density fluctuation reduces the mode conversion efficiency even at optimal angle considerably. Even though we do not have any experimental data on density fluctuation at the edge region in Heliotron $\mathrm{J}$ where the $\mathrm{O}-\mathrm{X}$ mode conversion takes place, the calculation suggests that the density fluctuation of $8 \%$ causes reduction in conversion efficiency by about $20 \%$. The density fluctuation effect is more significant as the density gradient is gentle, meaning that the steep density gradient is favorable for high $\mathrm{O}-\mathrm{X}$ mode conversion, which is typically observed in ECH plasmas in helical devices. 


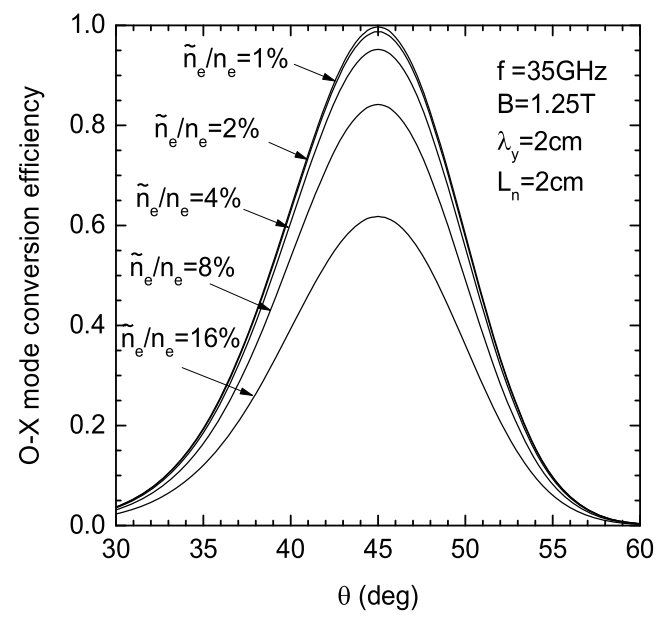

Fig. 5 Effect of density fluctuation on O-X mode conversion efficiency.

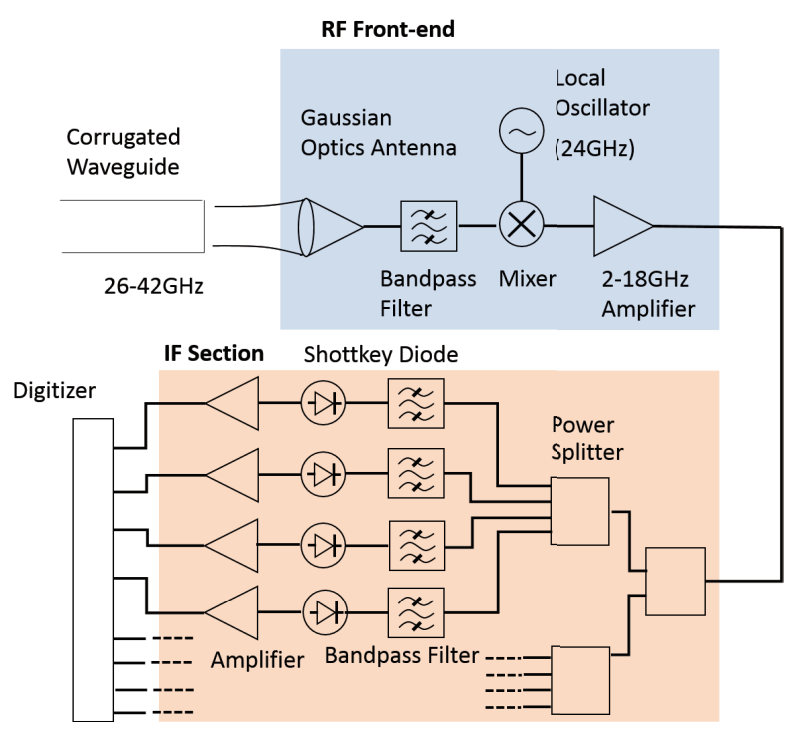

Fig. 6 Schematic of EBE radiometer system.

\section{EBE Diagnostic for Heliotron J}

An EBE diagnostic has been designed for Heliotron $\mathrm{J}$, based on the ray tracing calculation results. The designed EBE diagnostic consists of a steerable antenna, a waveguide transmission line and a heterodyne radiometer. A launcher and transmission line of corrugated waveguides for the existing $70 \mathrm{GHz}$ ECRH/ECCD system is applied for the EBE diagnostic instead of assembling a new independent system. A waveguide switch is installed $3 \mathrm{~m}$ before the launcher, which manually switches from the ECRH/ECCD to EBE measurement. A Gaussian-optics antenna, which can measure two cross-polarizations, is used between the corrugated waveguide and the Ka-band ( $24-42 \mathrm{GHz}$ ) rectangular waveguide. A SPDT PIN switch is set up in the Ka band rectangular waveguide line, which selects the orthogonal field components in a plasma discharge by applying an external bias voltage.

The heterodyne radiometer system has been assem-

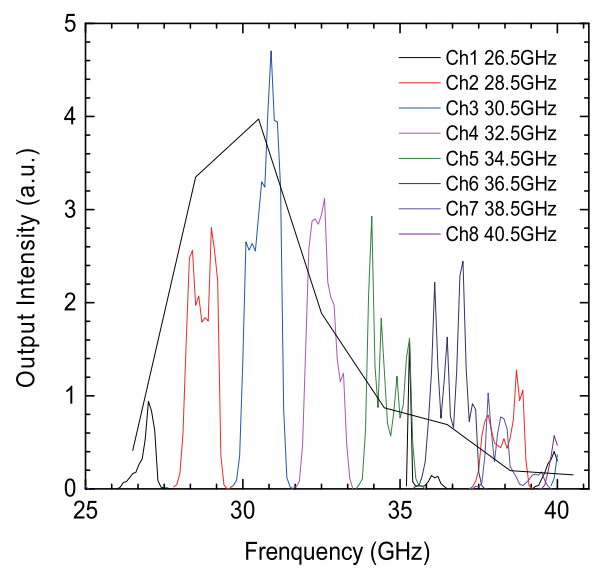

Fig. 7 Calibration Results of heterodyne radiometer. The black line denote the calibration results from a noise source, the others denote those from a frequency scanning microwave source.

bled and tested before installation on the Heliotron $\mathbf{J}$ device. Figure 6 shows a schematic of the radiometer, which covers Ka-band, corresponding to the cut-off density of $0.86-2.25 \times 10^{19} \mathrm{~m}^{-3}$. The radiometer consists of a $24 \mathrm{GHz}$ Gunn oscillator, a balanced mixer, a step attenuator, low-noise amplifiers, power dividers, band-pass filters, Shottky-barrier diodes. The band-pass filters of $1 \mathrm{GHz}$ band width allow tuning 8 channels of the radiometers to frequencies in the $26-41 \mathrm{GHz}$ range. The center frequencies of bandpass filters are $2.5 \mathrm{GHz}, 4.5 \mathrm{GHz}, 6.5 \mathrm{GHz}$, 8.5 GHz, $10.5 \mathrm{GHz}, 12.5 \mathrm{GHz}, 14.5 \mathrm{GHz}$ and $16.5 \mathrm{GHz}$.

Relative calibration has been performed with two kinds of microwaves sources as an input signal to the radiometer. One is a noise source of Ka-band (Hewlett Packard R347B, 26.5 - 40 GHz) which radiates broadband microwaves. The other is a frequency tunable microwave source of $26-40 \mathrm{GHz}$ frequency range, in which a powercalibrated microwaves of $12-20 \mathrm{GHz}$ generated by a solidstate signal generator (Vaunix LMS-203, 10 - 20 GHz), are doubled to $24-40 \mathrm{GHz}$ with an active frequency doubler (SAGE SFA-282SF-S1, 26.5-40 GHz). For the former source case, the radiometer output reflects the white broadband noise radiation input, and for the latter case, it reflects the discrete frequency radiation. Figure 7 shows the relative calibration results. It can be seen that both calibration results have the same tendency in output signal intensity, but not exactly the same performance due to the different characteristics between the two sources. Although the reason for fluctuations observed in the discrete frequency source is not clear, it may be related to the characteristics of the radiometer components such as mixer, low noise amplifiers, power splitter and band pass filter. The nW level of input microwaves signal is possible to detect beyond the noise level. Since the bandpass filters of $2.4 \mathrm{GHz}$ and $4.5 \mathrm{GHz}$ do not filter out high frequency components of $11-12 \mathrm{GHz}$ and $13.5-15 \mathrm{GHz}$ well, replacement into better ones is required. 


\section{Summary}

An EBE diagnostic system using B-X-O mode conversion process has been developed for determining electron temperature profiles at overdense plasmas in Heliotron J. The calculation results using the ray tracing codes, TRAVIS and KRAY, show that the current EC launcher antenna has an access window for the $\mathrm{O}-\mathrm{X}$ conversion under overdense plasma conditions. The ray tracing code, KRAY, which considers the 3-D magnetic configuration with finite beta, predicts that the radiation from the core region can be detected through the $\mathrm{B}-\mathrm{X}-\mathrm{O}$ mode conversion process. A multi-channel radiometer system has been designed, assembled and tested for EBE measurement. The EBE measurement in NBI plasmas will be performed in Heliotron $\mathrm{J}$ in the near future. Application of this radiometer system to the LHD device is also underway.

\section{Acknowledgments}

The authors are grateful to the Heliotron $\mathrm{J}$ staff for useful discussion. This work was performed with the support and under the auspices of the Collaboration Program of the Laboratory for Complex Energy Processes, IAE, Kyoto University, and the NIFS Collaborative Research Program (NIFS10KUHL030, NIFS13KUHL059), the NIFS/NINS project of Formation of International Network for Scientific Collaborations, and a Grant-in-Aid for Scientific Research, MEXT.

[1] H.P. Laqua, Plasma Phys. Control. Fusion 49, R1 (2007).

[2] H.P. Laqua et al., Phys. Rev. Lett. 78, 3467 (1997).

[3] H.P. Laqua et al., Phys. Rev. Lett. 81, 2060 (1998).

[4] F. Volpe et al., Rev. Sci. Instrum. 74, 1409 (2003).

[5] T. Obiki et al., Nucl. Fusion 41, 833 (2001).

[6] N.B. Marushchenko et al., Plasma Fusion Res. 2, S1129 (2007).

[7] E. Mjølhus, J. Plasma Phys. 31, 7 (1984).

[8] K. Nagasaki et al., Plasma Phys. Control. Fusion 44, 409 (2002).

[9] J. Preinhaelter and V. Kopeck'y, J. Plasma Phys. 10, 1 (1973). 